\title{
Forecasting Long Memory Series Subject to Structural Change: A Two-Stage Approach
}

\author{
Fotis Papailias $^{\mathrm{a}, \mathrm{b}, *}$, Gustavo Fruet Dias ${ }^{\mathrm{c}, \mathrm{d}, * *}$ \\ ${ }^{a}$ Queen's University Management School, Queen's University Belfast, UK \\ ${ }^{b}$ quantf Research, www. quantf.com \\ ${ }^{c}$ Department of Economics and Business, Aarhus University \\ ${ }^{d}$ CREATES, Aarhus University
}

\begin{abstract}
A two-stage forecasting approach for long memory time series is introduced. In the first step we estimate the fractional exponent and, applying the fractional differencing operator, we obtain the underlying weakly dependent series. In the second step, we perform the multi-step ahead forecasts for the weakly dependent series and obtain their long memory counterparts by applying the fractional cumulation operator. The methodology applies to stationary and nonstationary cases. Simulations and an application to seven time series provide evidence that the new methodology is more robust to structural change and yields good forecasting results.

Keywords: Forecasting, Spurious Long Memory, Structural Change, Local Whittle JEL classification: $\mathrm{C} 22, \mathrm{C} 53$
\end{abstract}

\section{Introduction}

The issue of analysing economic and other series which possess hyperbolically decaying autocorrelations has long been of concern in the time series analysis literature. The work of Granger (1980), Granger and Joyeux (1980) and Hosking (1981),

*Corresponding Author. Queen's University Management School, Queen's University Belfast, Riddel Hall, 185 Stranmillis Road, BT9 5EE, Northern Ireland. Tel.: +442890974667, Fax.: +442890974201. f.papailias@qub.ac.uk; f.papailias@quantf.com

** Gustavo Fruet Dias acknowledges support from CREATES, Center for Research in Econometric Analysis of Time Series (DNRF78), funded by the Danish National Research Foundation. 
among others, has been influential in the study and modelling of such long memory series; see Beran (1994) and Baillie (1996) for an extensive survey of this field.

There has been a major debate on the estimation of long memory series in both full and semi parametric setups, e.g. see, among others, Fox and Taqqu (1986), Sowell (1992), Robinson (1995), Beran et al. (1998), Robinson (2006), Shimotsu and Phillips (2006), Abadir, Distaso and Giraitis (2007) and Hualde and Robinson (2011) for more details.

However, the topic of forecasting long memory series is still growing. Diebold and Lindner (1996), Chan and Palma (1998), Bhansali and Kokoszka (2002), Bhardwaj and Swanson (2006) and Baillie et al. (2012), among others, have been concerned with predictions from ARFIMA models. A well-known approach is to obtain the predictions by using a truncated version of the infinite autoregressive representation of the model. Peiris (1987) and Peiris and Perrera (1988) discuss computationally feasible ways for calculating these predictions and Crato and Ray (1996) and Poskitt (2007) analyse information criteria in order to determine the lag of the autoregression.

In this paper, we suggest the use of a two-stage forecasting approach (TSF). The TSF is a simple and intuitive methodology. To begin with, we estimate the long memory parameter with any consistent estimator. Then we apply the fractional differencing operator which results in the underlying weakly dependent series. Finally, we compute the multi-step ahead forecasts for the latter and we apply the fractional cumulation operator ${ }^{1}$ in order to obtain the corresponding forecasts for the original long memory series. A similar approach has been adopted by Papailias et al. (2013) who are concerned with the bootstrapping of long memory series.

Our claim is that forecasts of the underlying weakly dependent series when translated to their long memory equivalents should provide, on average, smaller forecast errors, given that the weakly dependent series is less persistent, and hence, models are able to provide better forecasts. Therefore, TSF avoids any "loss" of information which might be the case when employing the truncation of the infinite AR representation of the model.

It should be noted that we are not concerned with the nature of the estimation of the series, i.e. full or semi parametric methods, and hence we do not discuss their

\footnotetext{
${ }^{1}$ This is the inverse of the fractional differencing operator.
} 
advantages and/or disadvantages. We rely simply on the consistency of the estimators to carry out our forecasting methodology. In our simulations and applications we use the Fully Extended Local Whittle (FELW) of Abadir, Distaso and Giraitis (2007), however other consistent estimators can be used equivalently.

A common issue that often arises when working with real time series which might exhibit long memory is the possibility of structural change. This is commonly referred to as spurious long memory. In such cases the change(s) in the structure of the series might be mistaken as long memory or it might even be a co-existence of strong dependence and structural change. This poses threats in the analysis and, consequently, forecasting of the series. Diebold and Inoue (2001) is among the first studies in the field that jointly analyse the phenomena of long memory and structural change and prove how structural change can be misinterpreted as long memory. Lazarovà (2005), Berkes et al. (2006), Ohanissian et al. (2008), Qu (2011), Shao (2011) and Iacone et al. (2013), among others, develop tests to accommodate the spurious long memory effect.

But, how should the applied researcher forecast series which might exhibit spurious long memory? What happens if the tests fail to distinguish between pure long memory and structural change? Wang et al. (2013) suggest that a simple autoregressive (AR) model should be used in the forecasting as it approximates well an ARFIMA process subject to a mean shift or a change in the long memory parameter.

In this paper we show via simulations that a simple AR model used in the second step of the hereby suggested TSF methodology results in accurate and more robust forecasts when applied to long memory series with a break in the mean or a change in the long memory parameter. This result is useful to practitioners who can employ the methodology even when there is a possibility of spurious long memory. An empirical exercise in seven real time series illustrates the applicability and advantages of the TSF methodology, and in some cases the truncated version of the infinite AR representation of the model, in an applied setup.

The rest of the paper is organised as follows: Section 2 provides some basic definitions and the algorithm of the proposed forecasting methodology. Section 3 introduces the structural change in long memory series and discusses the simulation results. Section 4 is concerned with the empirical exercise and Section 5 summarises the conclusions. 


\section{Long Memory: Concepts and Forecasting}

\subsection{Existing Framework}

We start by considering the following general fractionally integrated model

$$
(1-L)^{d} x_{t}=u_{t}, t=1, \ldots T
$$

where $L$ denotes the lag operator, $d$ is the degree of long memory and $u_{t}$ is a weakly dependent, or short-range dependent, process. Hence, in the above setup $x_{t}$ is $I(d)$ and $u_{t}$ is $I(0)$. We define $I(0)$ processes such that their partial sums converge weakly to Brownian motion; for more information regarding the definition of $I(0)$ processes see Stock (1994), Davidson and DeJong (2000), Davidson (2002), Müller (2008) and Davidson (2009) among others. We model $u_{t}$ as

$$
u_{t}=\psi(L) \varepsilon_{t}
$$

with $E\left(\varepsilon_{t}\right)=0, E\left(\varepsilon_{t}^{2}\right)=\sigma_{\varepsilon}^{2}$ and $E\left(\varepsilon_{t} \varepsilon_{s}\right)=0$ for all $t \neq s . \psi(\lambda)$ is given by $\psi(\lambda)=$ $\sum_{i=0}^{\infty} \psi_{i} \lambda_{i}$ where $\psi_{i}$ is a sequence of real numbers such that $\psi_{0}=1, \sum_{i=0}^{\infty}\left|\psi_{i}\right|<$ $\infty$ and $\sum_{i=0}^{\infty} \psi_{i} \lambda_{i} \neq 0$. In the case where $u_{t}$ follows a stationary and invertible $A R M A(p, q)$ model then $x_{t}$ becomes the widely known $\operatorname{ARFIMA}(p, d, q)$ model. For $|d|<0.5$ the process is stationary and invertible, whereas for $d>0.5$ the process is nonstationary. The above defined process belongs to the Type I fractionally integrated process; see Marinucci and Robinson (1999) and Robinson (2005) for definitions regarding nonstationary processes and Beran (1994) and Baillie (1996) for a more detailed introduction to long memory processes.

We can write $x_{t}$ as an infinite autoregression process as

$$
x_{t}=\sum_{i=1}^{\infty} \beta_{i} x_{t-i}+u_{t}
$$

where $\beta_{i}=\frac{\Gamma(i-d)}{\Gamma(i+1) \Gamma(d)}$ with $\Gamma(\cdot)$ being the gamma function. The above results follow from the definition of the fractional differencing operator, $(1-L)^{d}$, that is formally valid for any real $d$; see Hosking (1981) for more details.

The standard forecasting method in the literature suggests that, given knowledge of the parameters and using Equation (3), the theoretical s-step ahead forecast 
conditional on the available information at time $T$ is given by

$$
\widehat{x}_{T+s}=\sum_{i=1}^{\infty} \beta_{i} x_{T+s-i} .
$$

Assuming that $x_{T}$ can be modelled as an $\operatorname{ARFIMA}(\mathrm{p}, \mathrm{d}, \mathrm{q})$ process a feasible way to implement the above in practice is to estimate the parameters and use a truncated version of the autoregressive weights obtained from the canonical representation of the model. Then, the s-step ahead forecast is given by,

$$
\widehat{x}_{T+s}=\sum_{i=1}^{P} \widehat{\beta}_{i} x_{T+s-i} .
$$

Crato and Ray (1996) and Poskitt (2007) discuss different alternatives in choosing the optimal lag order $P$ such that Equation (4) is well approximated.

In a recent study Wang et al. (2013) suggest to fit an autoregressive model directly to the long memory series choosing the order, $k$, via a selection criterion, i.e. AIC, Akaike (1969), or, $C_{p}$, Mallows (1973). This results in,

$$
\widehat{x}_{T+s}=\sum_{i=1}^{k} \widehat{\beta}_{i}^{c} x_{T+s-i} .
$$

It is important to notice that $\widehat{\beta}_{i}$ and $\widehat{\beta}_{i}^{c}$ differ, because the former is a function of the parameters from the $\operatorname{ARFIMA}(\mathrm{p}, \mathrm{d}, \mathrm{q})$ specification computed as in Equation (3). Wang et al. (2013) conclude that AIC provides better results and hence it is also adopted in this study. Recently the topic of selection of long memory time series models has been analysed by Baillie et al. (2013).

\subsection{A Two-Stage Forecasting Algorithm}

Along the lines discussed in the previous subsection, we propose the use of a twostage forecasting methodology, TSF, to tackle the issue of forecasting long memory series. The general idea, first, is to obtain the forecasts for the underlying weakly dependent series. Secondly, by using the fractional cumulation operator, we obtain the corresponding forecasts for the long memory series. A similar approach has been 
applied to the bootstrap of such series in Papailias et al. (2013). The TSF procedure can be implemented in the following steps:

1. Estimate the long memory parameter (full or semi parametrically) and obtain $\widehat{d}$. Note that we assume $\widehat{d}$ to be a consistent estimator of $d$ with $(\widehat{d}-d)=$ $O_{p}\left(T^{-\delta}\right)$, with delta $>0$. Then, apply the differencing operator and obtain the weakly dependent process, $\eta_{t}$,

$$
\eta_{t}=(1-L)^{\widehat{d}} x_{t}=(1-L)^{\widehat{d}-d} u_{t}
$$

where obviously $\eta_{t}$ is $I(\widehat{d}-d)$.

2. Fit an $A R(P)$ to $\eta_{t}$. The 1 -step ahead forecast is given by

$$
\widehat{\eta}_{T+1}=\sum_{i=1}^{\widehat{P}} \widehat{\pi}_{i} \eta_{T+1-i}
$$

3. Expand the original weakly dependent series $\eta_{t}$ including the above forecast, thus $\widetilde{\eta}_{t}=\left(\eta_{1}, \ldots, \eta_{T}, \widehat{\eta}_{T+1}\right)^{\prime}$. Apply the fractional cumulation operator using $\widehat{d}$ and obtain the vector which includes the 1-step ahead forecast for the long memory series,

$$
\widetilde{x}_{t}=(1-L)^{-\widehat{d}} \widetilde{\eta}_{t}, t=1, \ldots, T, T+1 .
$$

The 1-step ahead forecast is then given by $\widetilde{x}_{T+1}$.

4. By continuing to repeat the above steps, we can recursively obtain the s-step ahead forecast for the long memory series, $\widetilde{x}_{T+s}$.

In what follows we use two choices for the lag $P$ : (i) one using the AIC, and (ii) using a high-order with respect to the sample size $\widehat{P}=\left\lfloor(\ln T)^{2}\right\rfloor ;\lfloor\cdot\rfloor$ denotes integer part.

The estimate $\widehat{d}$ can be obtained by MLE or a semi parametric method; see Fox and Taqqu (1986), Sowell (1992), Robinson (1995), Beran et al. (1998), Robinson (2006), Shimotsu and Phillips (2006) and Abadir, Distaso and Giraitis (2007) among others. For reasons of simplicity and robustness to possible model misspecifications we choose to use a semi parameteric method to obtain $\widehat{d}$, the Fully Extended Local Whittle (FELW) of Abadir, Distaso and Giraitis (2007). 
A simple definition of the FELW, which can be found in Abadir, Distaso and Giraitis (2011), is the following. Let there be $d \in(p-1 / 2, p+1 / 2]$, for $p=0,1,2, \ldots$. The periodogram of the $x_{t}$ series is defined as

$$
I^{F E L W}\left(\omega_{j}\right)=\left|1-e^{i \omega_{j}}\right|^{-2 p} I_{(1-L)^{d} y_{t}}\left(\omega_{j}\right)
$$

Then the FELW is obtained by minimising,

$$
R^{F E L W}(\cdot)=\ln \left[\frac{1}{m} \sum_{j=1}^{m} j^{2 d} I^{F E L W}\left(\omega_{j}\right)\right]-\frac{2 d}{m} \sum_{j=1}^{m} \ln (j) .
$$

The above estimator is consistent for stationary and nonstationary series. We use the common choice of $m=\left\lfloor T^{0.5}\right\rfloor$ bandwidth in the above estimation.

\section{Simulation Experiments}

As mentioned in the Introduction, a common issue that often arises in the analysis of real time series is the presence of structural change. This poses threats to the time series analysis as a series with a break in the mean might be mistaken as long memory and vice versa. Therefore, the use of a robust forecasting methodology is necessary to tackle the issue of spurious long memory. In this study we compare the predicting ability of the previously mentioned methods in simulated series with: (i) no structural change (pure long memory), (ii) a break in the mean of the series, (iii) a break in the long memory parameter. Therefore, we forecast the following processes,

$$
y_{t}= \begin{cases}\mu+x_{t}, & 1 \leq t \leq b \\ \mu+\Delta+x_{t}, & b+1 \leq t \leq T\end{cases}
$$

and

$$
y_{t}= \begin{cases}\mu+x_{t}^{d_{1}}, & 1 \leq t \leq b \\ \mu+x_{t}^{d_{2}}, & b+1 \leq t \leq T\end{cases}
$$

with $\mu$ being the mean of the series, $x_{t}^{d_{1}}=(1-L)^{-d_{1}} u_{t}$ and $x_{t}^{d_{2}}=(1-L)^{-d_{2}} u_{t}$ for $d_{1} \neq d_{2}$. Obviously if in the case of Equation (12) $\Delta=0$ or in the case of Equation 
(13) $d_{1}=d_{2}$ there is no structural change. For reasons of simplicity we choose to use only one break in the mean or in the long memory parameter.

We report results from the following DGPs:

- Case 1: $\operatorname{ARFIMA}(0, \mathrm{~d}, 0)$ using Equation (12) with $d=\{0,0.2,0.4\}, T=\{128$, $256,512\}, b=0$ (no break) and $b=0.5 T$ with $\Delta .=1.5$ (Table 1 ),

- Case 2: $\operatorname{ARFIMA}(1, \mathrm{~d}, 0)$ using Equation (12) with $\phi=0.5, d=\{0,0.2,0.4\}$, $T=\{128,256,512\}, b=0$ (no break) and $b=0.5 T$ with $\Delta .=1.5$ (Table 2),

- Case 3: $\operatorname{ARFIMA}(1, \mathrm{~d}, 0)$ using Equation (13) with $\phi=\{0,0.5\}, T=512$, $b=0.5 T$ and $\left(d_{1}, d_{2}\right)=\{(0,0.4),(0.4,0),(0.2,0.4),(0.4,0.2)\}$ (Table 3$)$.

It should be noted that we do not experiment with nonstationary series as the structural break tests introduced in the empirical section of the paper are designed for stationary series only. For all Cases we report the results when there is no break $(b=0)$ and when a break occurs at the half of the sample. More results for different break points $(b=0.25 T, b=0.75 T)$ are available on request, however the qualitative conclusion does not change. Finally, the results for $T=1024$ are omitted for two reasons: (i) the qualitative conclusion does not change compared to $T=512$ and (ii) to mimic the conditions faced by the applied research who often deals with limited data availability.

For each series realisation we compute the 1 to 12 -step ahead forecasts using the following methodologies:

- $A R(A I C)$ : fit a simple autoregressive model with order chosen by the AIC as in Equation (6) and Wang et al. (2013),

- $A R(P)$ : fit a simple autoregressive model with order $\widehat{P}=\left\lfloor(\ln T)^{2}\right\rfloor$ and proceed as above,

- $A R(A I C)$-Trunc.: truncate the infinite summation up to a specific lag chosen by the AIC as in Equation (5),

- $A R(P)-$ Trunc.: truncate the infinite summation up to lag $\widehat{P}=\left\lfloor(\ln T)^{2}\right\rfloor$ and proceed as above, 
- $A R(A I C)-T S F$ : use the TSF algorithm and fit an autoregressive model with order chosen by AIC to the underlying weakly dependent process,

- $A R(P)-T S F$ : use the TSF algorithm and fit an autoregressive model with order $\widehat{P}=\left\lfloor(\ln T)^{2}\right\rfloor$ to the underlying weakly dependent process.

Then, we count which method results in the smallest squared forecast error ${ }^{2}$. This process is repeated for $\mathrm{R}=1000$ series realisations according to the relevant DGP. For reasons of brevity we report $h=\{1,3,6,12\}$ steps ahead.

Starting with the left panels of Table 1 we see that in the case of no break, thus simple (fractional) white noise, $\mathrm{AR}(\mathrm{AIC})$ produces the smallest forecast error for all steps-ahead. The next best method seems to be AR(AIC)-TSF. This result is expected given the simplicity of the process.

However, if we look at the results of series with a break in the mean, on the right panel of Table 1 , we see that $\mathrm{AR}(\mathrm{AIC})-\mathrm{TSF}$ and AR(P)-TSF produce the best results with the TSF methods producing the smallest forecast error for more than $40 \%$ of the times across all forecast horizons. This result is very encouraging and provides a first evidence on the robustness of the TSF methodology. Of course, we have to ask ourselves why this is the case. At first, it is easy to understand that simple AR models (incl. high order models) fail to produce accurate forecasts in the presence of a break. Next, we see that the truncated versions of the AR expansion produce moderate results and, in that case, the $\mathrm{AR}(\mathrm{P})$-Trunc. is better. This leads to the fact that the break is mistaken as long memory.

But what does TSF do and results in more accurate forecasts? In the first step of the TSF we estimate the "long memory" and we fractionally filter the series. This means that the break presence is "smoothed" in the filtered series where the simple AR models can produce better forecasts. This is the key aspect of the TSF methodology that makes it favourable compared to its competitors. As we move to DGPs with long memory and a break in the mean we see that TSF methods are better, on average, followed by the simple AR methods. This result across all sample sizes.

\footnotetext{
${ }^{2}$ The squared forecast error for the s-step ahead forecast is defined as $\left(y_{T+s}-\widehat{y}_{T+s}\right)^{2}$ where $y_{T+s}$ is the true value and $\widehat{y}_{T+s}$ is the forecast estimate.
} 
Investigating the ARFIMA(1,d,0) DGP with $\phi=0.5$ in Table 2 we see that the previously discussed qualitative conclusion still holds and the evidence in favour of the TSF is even stronger. Now, we also find cases with series without break, i.e. pure long memory, where the TSF methodology works better. In the medium and large sample sizes, $T=256$ and $T=512$, the AR(AIC)-TSF is better compared to all other methods providing a smaller forecast error in more than $20 \%$ of the times. In the cases of spurious long memory with a break in the mean we see that AR(AIC)-TSF is still better, on average, across all sample sizes, values of $d$ and forecast horizons.

The so far analysis is concerned with a break in the mean of the series. Table 3 reports the results when there is a break in the long memory parameter. Here we see that $\mathrm{AR}(\mathrm{AIC})$ seems to be the best choice of method as it produces the smallest forecast error more than $20 \%$ of the times followed by the AR(AIC)-TSF and the AR(P)-TSF. However, if we sum the number of times the simple methods, i.e. $\mathrm{AR}(\mathrm{AIC})+\mathrm{AR}(\mathrm{P})$, and the TSF methods, i.e. $\mathrm{AR}(\mathrm{AIC})-\mathrm{TSF}+\mathrm{AR}(\mathrm{P})-\mathrm{TSF}$, produce the smallest forecast error we see that the TSF methods are better in the majority of cases (with a success rate of more than 40\%).

Therefore, now that we have enough evidence and a better understanding of the reasons that $\mathrm{AR}(\mathrm{AIC})$ and $\mathrm{AR}(\mathrm{AIC})$-TSF result in better performance, a question to consider is what happens if we exclude all other methods in the simulation comparison. This is what we do in Table 4 . Table 4 reports the results for all cases analysed above using series with a break in the mean or the long memory parameter with the two competing methods. In the cases of a break in the mean (top panel of Table 4) we see that AR(AIC)-TSF is a clear winner and it should always be chosen. However, looking at the bottom panel of the table we see that AR(AIC)-TSF is better in the cases where there is an increase in the long memory parameter after the break, i.e. $(0,0.4)$ and $(0.2,0.4)$ cases.

From the above simulations we can conclude that the hereby suggested TSF methodology is more robust to structural change and provides better forecasts if breaks occur in the mean or the long memory parameter. Therefore it should be chosen by the applied researcher who is often unaware of the true nature of the series, i.e. pure or spurious long memory. 


\section{Empirical Exercise}

In the last part of this study we examine the applicability of the TSF methodology in real time series. We report results for seven series where the distinction between pure and spurious long memory is difficult. These include four US macroeconomic series, the German CPI inflation, the UK M1 and the seasonally adjusted monthly temperatures for the northern hemisphere. In details:

- Series 1-4: US Macroeconomic series. Monthly observations of NAPM Production Index, Employees on Non-farm Payrolls - Total Private, Employee Hours in Non-ag. Establishments (AR, bil. hours), NAPM New Orders Index. These are included in the well known Stock and Watson (2006) dataset. The data was collected from the authors' website and has been transformed appropriately as in Stock and Watson (2006). It spans from 1960-02-01 to 2003-12-01 (527 observations).

- Series 5: DE CPI inflation. Period-to-period monthly change of the German CPI. The data was collected using Macrobond Financial Software and spans from 1991-01-01 to 2013-02-01 (266 observations).

- Series 6: UK M1. Period-to-period monthly change of the UK M1. The data was collected using Macrobond Financial Software and spans from 1986-09-01 to 2013-02-01 (318 observations).

- Series 7: Climate Series. Seasonally Adjusted Monthly Temperatures for the Northern Hemisphere (in degrees Centigrade, as a deviation from the monthly averages) for the Northern Hemisphere. This data is chosen because it has been extensively analysed in the literature; see Smith (1993), Beran (1994), Deo and Hurvich (1998), Shao (2011). The series was collected from C. M. Hurvich's website. It spans from 1854-1989 (1632 observations).

In the above cases we try to think from the applied researcher's point of view and perform a rolling forecasting exercise which tends to be more robust to structural changes. We compute the 1- to 12- step ahead forecasts for an out-of-sample crossvalidation period of $N=96$ observations (i.e. 8 years) for all series apart from Series 7 where we use 120 observations (i.e. 10 years) due to data availability. The length 
of the window in each case is given by $\left(T-N-h_{\max }+1\right)$ where $h_{\max }=12$. At the end of the forecasting exercise we collect $N$ forecasts for all forecast horizons using a simple $\mathrm{AR}(1)$ model and the previously analysed $\mathrm{AR}(\mathrm{AIC}), \mathrm{AR}(\mathrm{P}), \mathrm{AR}(\mathrm{AIC})$ Trunc., AR(P)-Trunc., AR(AIC)-TSF and AR(P)-TSF. We compare the predicting ability of the models by reporting the Root Mean Squared Forecast Error (RMSFE) ${ }^{3}$ of each model relative to the $\mathrm{AR}(1)$ which acts as a simple benchmark. Of course, $\mathrm{AR}(1)$ serves as a reference point which still allows for the inter-comparison of the other methods.

In Table 5 we report the FELW estimates along with the results of two tests for a break in the mean of the series. At first we apply the average F-type test (aveF) ${ }^{4}$ as in Andrews (1993) and Andrews and Ploberger (1994) with p-values obtained as in Hansen (1997). This is a simple test and does not account for long memory. The second test we employ is introduced in Shao (2011) and tests for a break in the mean of the series in the presence of long memory. However, it must be noted that the test is valid only for stationary cases. The p-values are interpolated from Table 1 in Shao (2011). The null hypothesis for both tests is "no structural change" and the significance level is $90 \%$. For reasons of brevity we report $h=\{1,3,6,12\}$ steps ahead.

In Table 5 we see that Shao (2011) test rejects the null hypothesis in all series indicating that there is a break in the mean. In most cases this is accompanied by a large degree of long memory (apart from Series 1 and Series 4 where $\widehat{d}$ is 0.04 and 0.063 respectively). This is inline with the aveF test which also rejects the null hypothesis in all series apart from Series 3.

A first glance at Table 6 shows that the worst performers are the simple AR(AIC) and $\mathrm{AR}(\mathrm{P})$ across all series. Analysing Series 1 we see that the AR(AIC)-TSF provides the smallest forecast error across all forecasting horizons followed by the AR(AIC)-Trunc. This fact is not surprising based on the simulation discussion of the previous section.

\footnotetext{
${ }^{3}$ The RMSFE for the s-step ahead forecast across $N$ evaluation periods is defined as $\sqrt{\frac{1}{N} \sum\left(y_{T+s}-\widehat{y}_{T+s}\right)^{2}}$ where $y_{T+s}$ is the true value and $\widehat{y}_{T+s}$ is the forecast estimate.

${ }^{4}$ The supF and exponential, expF, produce almost identical results and are omitted. This part of computations is based on the $\mathrm{R}$ package "strucchange".
} 
For Series 2 and 3 we observe minor differences between AR(AIC)-Trunc. and $\mathrm{AR}(\mathrm{AIC})-\mathrm{TSF}$, with the latter being superior to the rest of the methods. For Series 4 and 5 we see that AR(AIC)-TSF is slightly better in shorter horizons and for Series 6 we see that AR(AIC)-TSF produces the smallest forecast error across all horizons.

For the last case of Series 7 we observe that all methods perform similarly. This might be explained by the fact that there is plenty of data available and the window length is much larger resulting in better estimation and more accurate forecasting. In shorter horizons, $h=1$ and $h=3$, we see that AR(AIC)-TSF performs slightly better than the other methods however in longer horizons, $h=6$ and $h=12$, we see that $\mathrm{AR}(\mathrm{AIC})$-Trunc. and $\mathrm{AR}(\mathrm{P})$-Trunc. provide the forecasts with the smallest error.

The empirical results in conjunction with the simulation results point out that the truncated and the TSF methods should be considered in practical analysis with the TSF being more robust in breaks in the mean and the long memory parameter.

\section{Concluding Remarks}

In this paper, we investigate the topic of forecasting long memory time series focusing on spurious long memory cases. We introduce a two-stage approach, which consists of applying the fractional differencing operator using any consistent estimate of the long memory parameter to retrieve the underlying short memory series. Then we obtain forecasts of this weakly dependent series in the usual way and, using the fractional cumulation operator, we obtain the corresponding forecast estimates for the strongly dependent series.

A detailed simulation study suggests that the new methodology is robust to breaks in the mean or the long memory parameter and should be employed by the applied researcher who is unaware of the true structure of the data. The empirical exercise provides evidence that the two-stage approach and, in some cases, the truncated version of the canonical AR representation of the model. However, as shown by the simulation study in this paper, the latter is not suggested when breaks occur in the memory parameter.

The issues of multiple breaks, breaks in the short-run dynamics and/or joint breaks in the mean, long memory and short-run dynamics are not covered here and 
should be further investigated as there exist a large number of real time series with frequent changes in the structure. 
Abadir, M. K., Distaso, W., \& Giraitis, L. (2007). Nonstationarity-extended local whittle estimation. Journal of Econometrics, 141, 1353-1384.

Abadir, M. K., Distaso, W., \& Giraitis, L. (2011). An $I(d)$ model with trend and cycles. Journal of Econometrics, 163, 186-199.

Akaike, H. (1969). Power spectrum estimation through autoregressive model fitting. Annals of Institute of Statistics Mathematics, 21, 407419.

Andrews, D. W. K. (1993). Tests for parameter instability and structural change with unknown change point. Econometrica, 61, 821-856.

Andrews, D. W. K., \& Ploberger, W. (1994). Optimal tests when a nuisance parameter is present only under the alternative. Econometrica, 62, 1384-1414.

Baillie, R. T. (1996). Long memory and fractional integration in econometrics. Journal of Econometrics, 73, 5-59.

Baillie, R. T., Kapetanios, G., \& Papailias, F. (2013). Modified information criteria and selection of long memory time series models. Computational Statistics \& Data Analysis, 76, 116-131.

Baillie, R. T., Kongcharoen, C., \& Kapetanios, G. (2012). Prediction from ARFIMA models: Comparisons between MLE and semiparametric estimation procedures. International Journal of Forecasting, 28, 46-53.

Beran, J. (1994). Statistics for Long Memory Processes. Chapman \& Hall.

Beran, J., Bhansali, R. J., \& Ocker, D. (1998). On unified model selection for stationary and nonstationary short- and long-memory autoregressive series. Biometrika, 85, 921-934.

Berkes, I., Horváth, L., Kokoszka, P., \& Shao, Q.-M. (2006). On discriminating between long-range dependence and changes in mean. Annals of Statistics, 34, 1140-1165.

Bhansali, R. J., \& Kokoszka, P. S. (2002). Computation of the forecast coefficients for multistep prediction of long-range dependent time series. International Journal of Forecasting, 18, 181-206. 
Bhardwaj, G., \& Swanson, N. R. (2006). An empirical investigation of the usefulness of ARFIMA models for predicting macroeconomic and financial time series. Journal of Econometrics, 131, 539-578.

Chan, N. H., \& Palma, W. (1998). State space modeling of long memory processes. Annals of Statistics, 26, 719-740.

Crato, N., \& Ray, B. (1996). Model selection and forecasting of long-range dependent processes. Journal of Forecasting, 18, 181-206.

Davidson, J. (2002). Establishing conditions for the functional central limit theorem in nonlinear and semiparametric time series processes. Journal of Econometrics, 106, 243-269.

Davidson, J. (2009). When is a time series I(0)? In The Methodology and Practice of Econometrics: A Festschrift in Honour of David F. Hendry, Castle, J. L., \& Shephard, N., eds. Oxford University Press.

Davidson, J., \& DeJong, R. (2000). The functional central limit theorem and convergence to stochastic integrals II: fractionally integrated processes. Econometric Theory, 16, 621-642.

Deo, R. S., \& Hurvich, C. M. (1998). Linear trend with fractionally integrated errors. Journal of Time Series Analysis, 19, 379-397.

Diebold, F.X., \& Inoue, A. (2001). Long memory and regime switching. Journal of Econometrics, 105, 131159.

Diebold, F. X., \& Lindner, P. (1996). Fractional integration and interval prediction. Economics Letters, 50, 305-313.

Fox, C., \& Taqqu, M. S. (1986). Large sample properties of parameter estimates for strongly dependent processes. Annals of Statistics, 14, 517-532.

Granger, C. W. J. (1980). Long memory relationships and the aggregation of dynamic models. Journal of Econometrics, 14, 227-238. 
Granger, C. W. J., \& Joyeux, R. (1980). An introduction to long memory time series models and fractional differencing. Journal of Time Series Analysis, 1, 15-39.

Hansen, B. E. (1997). Approximate asymptotic p values for structural-change tests. Journal of Business 8 Economics Statistics, 15, 60-67.

Hosking, J. R. M. (1981). Fractional differencing. Biometrika, 68, 165-176.

Hualde, J., \& Robinson, P. M. (2011). Gaussian pseudo-maximum likelihood estimation of fractional time series models. Annals of Statistics, 39, 3152-3181.

Iacone, F., Leybourne, S. J., \& Taylor, A. M. R. (2013). A fixed-b test for a break in level at an unknown time under fractional integration. Journal of Time Series Analysis, 35, 40-54.

Lazarovà, S. (2005). Testing for structural change in regression with long memory processes. Journal of Econometrics, 129, 329-372.

Mallows, C. L. (1973). Come comments on $C_{p}$. Technometrics, 15, 661675.

Marinucci, D., \& Robinson, P. M. (1999). Alternative forms of fractional Brownian motion. Journal of Statistical Planning and Inference, 80, 111122.

Müller, U. K. (2008). The impossibility of consistent discrimination between $\mathrm{I}(0)$ and I(1) processes. Econometric Theory, 24, 616-630.

Ohanissian, A., Russell, J. R., \& Tsay, R. S. (2008). True or spurious long memory? A new test. Journal of Business 85 Economics Statistics, 26, 161-175.

Papailias, F., Kapetanios, G., Taylor, A. M. R. (2013). A fractional differencing bBootstrap for long memory processes. Unpublished Manuscript. A previous version of the paper has been circulated as: Kapetanios, G., Papailias, F., (2011). Block bootstrap and long memory. School of Economics and Finance, Queen Mary, University of London, Working Paper No. 679.

Peiris, M. S. (1987). A note on the predictors of difference sequences. The Australian Journal of Statistics, 29, 42-48. 
Peiris, M. S., \& Perrera, B. J. C. (1988). On prediction with fractionally differenced ARMA models. Journal of Time Series Analysis, 9, 215-220.

Poskitt, D. S. (2007). Autoregressive approximation in nonstandard situations: the fractionally integrated and non-invertible cases. Annals of the Institute of Statistical Mathematics, 59, 697-725.

Qu, Z. (2011). A test against spurious long memory. Journal of Business $\& 5$ Economic Statistics, 29, 423-438.

Robinson, P. M. (1995). Gaussian semi-parametric estimation of long range dependence. Annals of Statistics, 23, 1630-1661.

Robinson, P. M. (2005). The distance between rival nonstationary fractional processes. Journal of Econometrics, 128, 283-300.

Robinson, P. M. (2006). Conditional-sum-of-squares estimation of models for stationary time series with long memory. JIMS Lecture Notes-Monograph Series: Time Series and Related Topics, 52, 130-137.

Shao, X. (2011). A simple test of changes in mean in the possible presence of longrange dependence. Journal of Time Series Analysis, 32, 598-606.

Shimotsu, K., \& Phillips, P. C. B. (2006). Local whittle estimation and some of its variants. Journal of Econometrics, 130, 209-233.

Smith, R. (1993). Long-range dependence and global warming. In: Statistics for the Environment, Barnett, V., \& Turkman, K. F., eds. New York: Wiley.

Sowell, F. B. (1992). Maximum likelihood estimation of stationary univariate fractionally integrated time series models. Journal of Econometrics, 53, 165-188.

Stock, J. H. (1994). Deciding between I(1) and I(0). Journal of Econometrics, 63, 105-131.

Stock, J. H., \& Watson, M. W. (2006). Handbook of Economic Forecasting, 1, 515554. Elsevier. 
Wang, C. S.-H., Bauwens, L., \& Hsiao, C. (2013). Forecasting a long memory process subject to structural breaks. Journal of Econometrics, 177, 171-184.

Wright, J. H. (1995). Stochastic orders of magnitude associated with two-stage estimators of fractional arima systems. Journal of Time Series Analysis, 16-1, 119-126. 


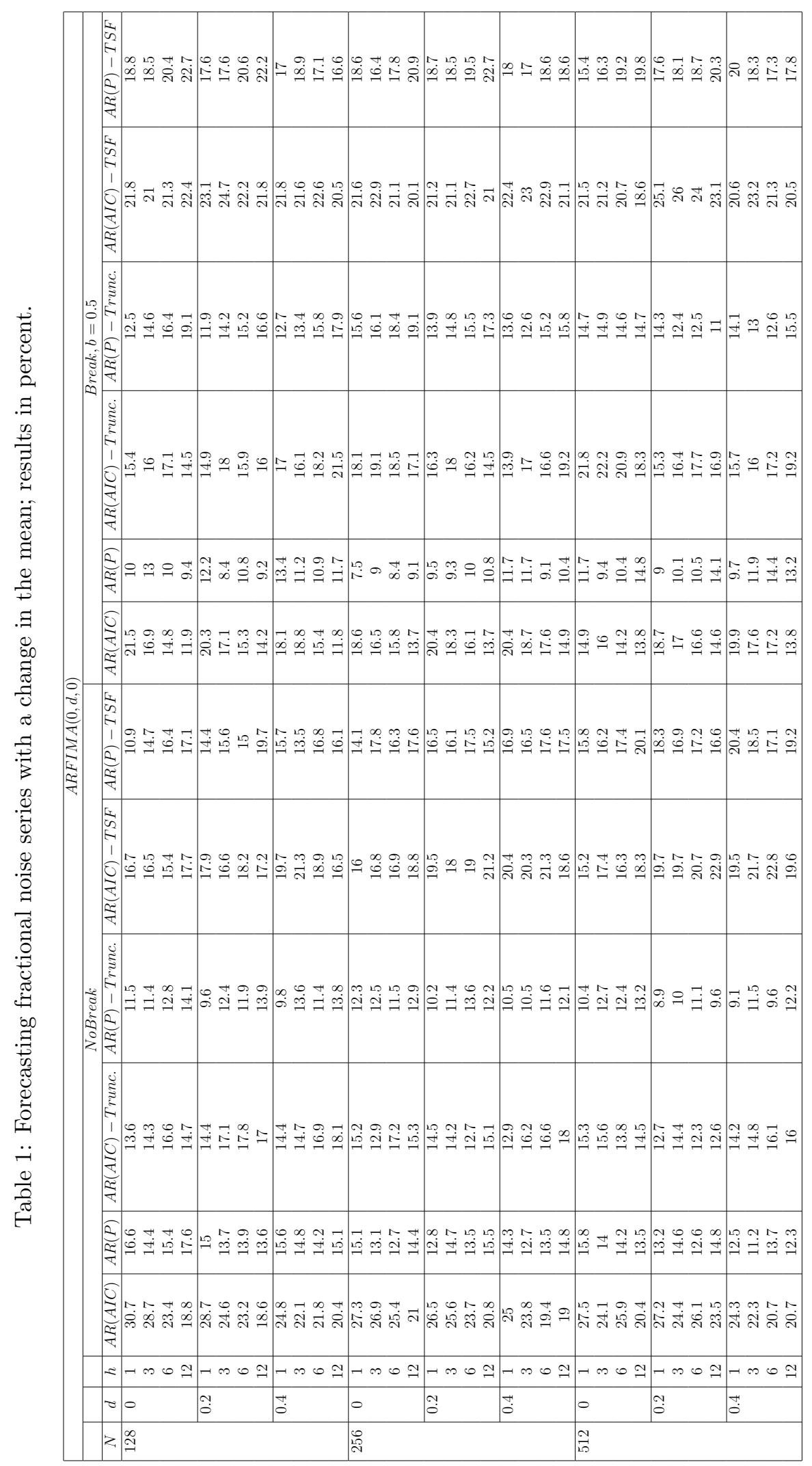




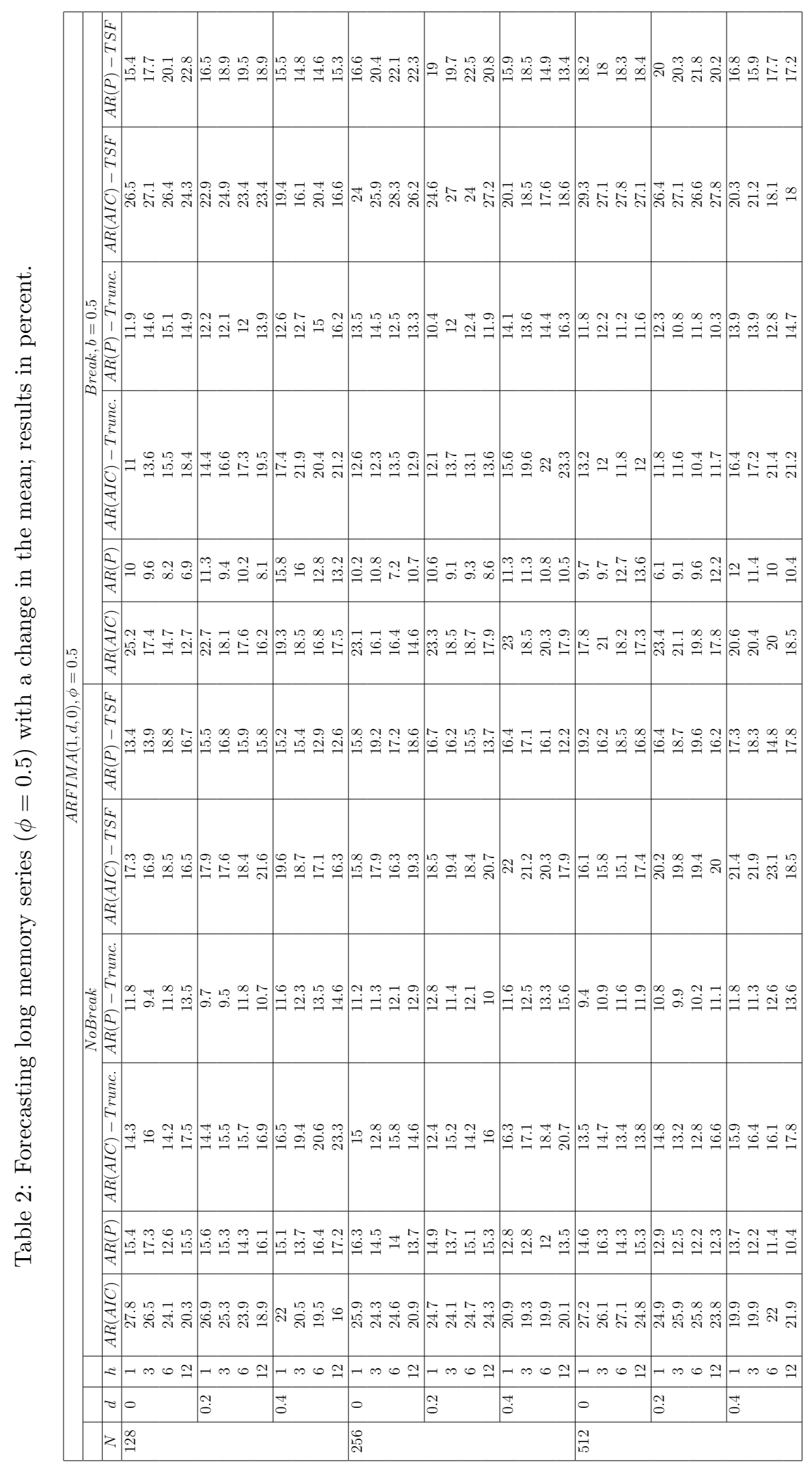




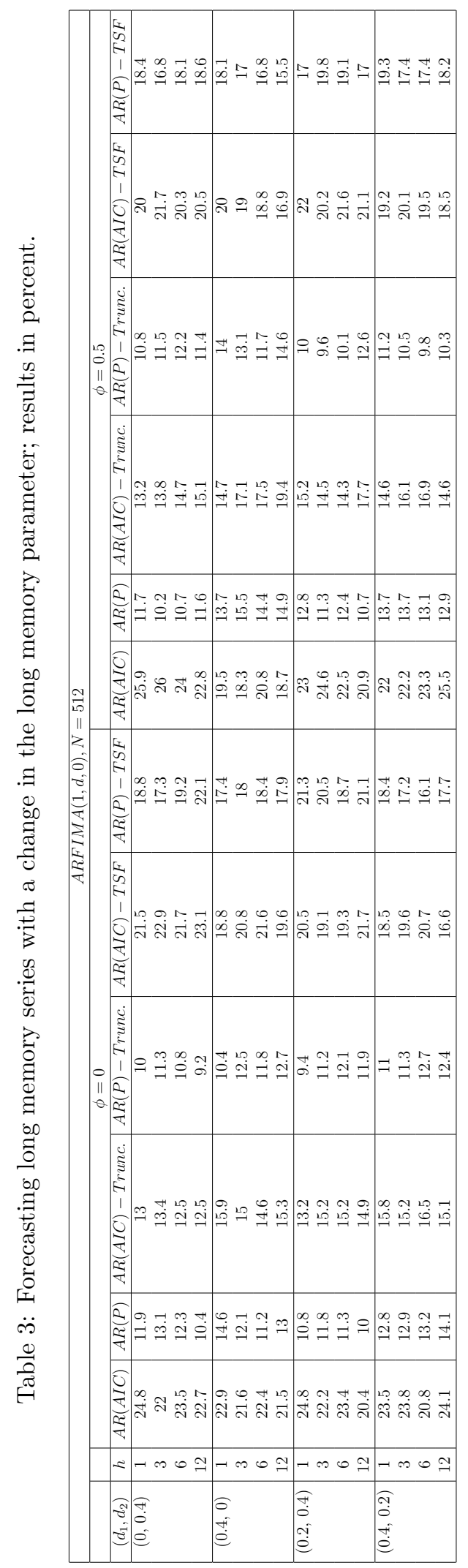


Table 4: Comparing two of the methods; results in percent.

\begin{tabular}{|c|c|c|c|c|c|}
\hline \multicolumn{6}{|c|}{$A R F I M A(1, d, 0), N=512, b=0.5$} \\
\hline & & \multicolumn{2}{|c|}{$\phi=0$} & \multicolumn{2}{|r|}{$\phi=0.5$} \\
\hline$d$ & $h$ & $A R(A I C)$ & $A R(A I C)-T S F$ & $A R(A I C)$ & $A R(A I C)-T S F$ \\
\hline \multirow[t]{4}{*}{0} & 1 & 46.6 & 53.4 & 42.3 & 57.7 \\
\hline & 3 & 43.7 & 56.3 & 40.4 & 59.6 \\
\hline & 6 & 46.1 & 53.9 & 36 & 64 \\
\hline & 12 & 47.4 & 52.6 & 36.6 & 63.4 \\
\hline \multirow[t]{4}{*}{0.2} & 1 & 45.3 & 54.7 & 42.1 & 57.9 \\
\hline & 3 & 46.4 & 53.6 & 40.7 & 59.3 \\
\hline & 6 & 44.3 & 55.7 & 39.7 & 60.3 \\
\hline & 12 & 47.1 & 52.9 & 36.6 & 63.4 \\
\hline \multirow[t]{4}{*}{0.4} & 1 & 47.9 & 52.1 & 48.9 & 51.1 \\
\hline & 3 & 49.4 & 50.6 & 49.9 & 50.1 \\
\hline & 6 & 49.8 & 50.2 & 50.4 & 49.6 \\
\hline & 12 & 49.6 & 50.4 & 54.5 & 45.5 \\
\hline$\left(d_{1}, d_{2}\right)$ & $h$ & $A R(A I C)$ & $A R(A I C)-T S F$ & $A R(A I C)$ & $A R(A I C)-T S F$ \\
\hline \multirow[t]{4}{*}{$(0,0.4)$} & 1 & 49 & 51 & 46.1 & 53.9 \\
\hline & 3 & 48.5 & 51.5 & 46 & 54 \\
\hline & 6 & 47.4 & 52.6 & 45 & 55 \\
\hline & 12 & 46.7 & 53.3 & 46.3 & 53.7 \\
\hline \multirow[t]{4}{*}{$(0.4,0)$} & 1 & 52 & 48 & 52.3 & 47.7 \\
\hline & 3 & 52.8 & 47.2 & 57.5 & 42.5 \\
\hline & 6 & 54.4 & 45.6 & 62.7 & 37.3 \\
\hline & 12 & 54.9 & 45.1 & 64.9 & 35.1 \\
\hline \multirow[t]{4}{*}{$(0.2,0.4)$} & 1 & 48.2 & 51.8 & 48.5 & 51.5 \\
\hline & 3 & 45.5 & 54.5 & 48.4 & 51.6 \\
\hline & 6 & 46 & 54 & 48.5 & 51.5 \\
\hline & 12 & 45.1 & 54.9 & 47.6 & 52.4 \\
\hline \multirow[t]{4}{*}{$(0.4,0.2)$} & 1 & 51.8 & 48.2 & 51.6 & 48.4 \\
\hline & 3 & 50.7 & 49.3 & 54.1 & 45.9 \\
\hline & 6 & 53.6 & 46.4 & 56.1 & 43.9 \\
\hline & 12 & 57.8 & 42.2 & 60.3 & 39.7 \\
\hline
\end{tabular}


Table 5: Various statistics for seven real time series.

\begin{tabular}{|c|c|c|c|c|}
\hline Series & $\widehat{d}$ & Shao (2011) Critical & Shao (2011) Statistic & aveF \\
\hline 1 & 0.040 & 29.935 & 32.255 & 0.000 \\
\hline 2 & 0.250 & 36.509 & 39.237 & 0.005 \\
\hline 3 & 0.242 & 36.317 & 39.384 & 0.319 \\
\hline 4 & 0.063 & 30.660 & 31.225 & 0.000 \\
\hline 5 & 0.455 & 42.476 & 100.738 & 0.000 \\
\hline 6 & 0.165 & 33.849 & 40.044 & 0.003 \\
\hline 7 & 0.484 & 43.748 & 76.275 & 0.000 \\
\hline $\begin{array}{l}\text { hours), } \\
\text { M1 UK, } \\
\text { Hemisph }\end{array}$ & $\begin{array}{l}\text { ies } 7 \text { : } \\
\text {. Leve }\end{array}$ & $\begin{array}{l}\text { uction Index, Series } \\
\text { 3: Employee Hours i } \\
\text { M New Orders Index } \\
\text { sonally Adjusted Mon } \\
\text { Significance: } 90 \%\end{array}$ & $\begin{array}{l}\text { Employees on Non-far } \\
\text { Non-ag. establishment } \\
\text { Series 5: CPI Germany } \\
\text { ly Temperatures for the }\end{array}$ & $\begin{array}{l}\text { Payrolls } \\
\text { AR, bil } \\
\text { Series } 6 \\
\text { Northern }\end{array}$ \\
\hline
\end{tabular}


Table 6: Forecasting results for seven real time series.

\begin{tabular}{|c|c|c|c|c|c|c|c|c|}
\hline & $h=1$ & $h=3$ & $h=6$ & $h=12$ & $h=1$ & $h=3$ & $h=6$ & $h=12$ \\
\hline & \multicolumn{4}{|c|}{ Series 1} & \multicolumn{4}{|c|}{ Series 2} \\
\hline$A R(A I C)$ & 1.542 & 1.616 & 1.653 & 1.672 & 0.804 & 0.687 & 0.764 & 0.971 \\
\hline$A R(P)$ & 1.961 & 2.061 & 2.104 & 2.160 & 0.964 & 0.914 & 1.149 & 1.494 \\
\hline$A R(A I C)-$ Trunc. & 0.659 & 0.603 & 0.621 & 0.694 & 0.761 & 0.618 & 0.632 & 0.778 \\
\hline$A R(P)-$ Trunc. & 0.720 & 0.690 & 0.737 & 0.869 & 0.860 & 0.760 & 0.892 & 1.171 \\
\hline$A R(A I C)-T S F$ & 0.624 & 0.568 & 0.594 & 0.721 & 0.765 & 0.635 & 0.657 & 0.799 \\
\hline \multirow[t]{2}{*}{$A R(P)-T S F$} & 0.653 & 0.624 & 0.733 & 0.966 & 0.815 & 0.700 & 0.770 & 0.955 \\
\hline & \multicolumn{4}{|c|}{ Series 3} & \multicolumn{4}{|c|}{ Series 4} \\
\hline$A R(A I C)$ & 0.936 & 0.964 & 1.000 & 0.992 & 1.393 & 1.504 & 1.510 & 1.525 \\
\hline$A R(P)$ & 1.028 & 1.050 & 1.086 & 1.137 & 1.793 & 1.992 & 2.008 & 2.087 \\
\hline$A R(A I C)-$ Trunc. & 0.918 & 0.961 & 1.004 & 0.982 & 0.709 & 0.625 & 0.633 & 0.708 \\
\hline$A R(P)-$ Trunc. & 0.975 & 1.025 & 1.038 & 1.046 & 0.731 & 0.671 & 0.695 & 0.823 \\
\hline$A R(A I C)-T S F$ & 0.920 & 0.963 & 1.007 & 0.978 & 0.700 & 0.612 & 0.636 & 0.795 \\
\hline \multirow{2}{*}{$A R(P)-T S F$} & 0.968 & 1.023 & 1.034 & 1.023 & 0.694 & 0.621 & 0.664 & 0.884 \\
\hline & \multicolumn{4}{|c|}{ Series 5} & \multicolumn{4}{|c|}{ Series 6} \\
\hline$A R(A I C)$ & 0.971 & 0.968 & 0.960 & 1.018 & 1.000 & 1.000 & 1.003 & 1.004 \\
\hline$A R(P)$ & 1.196 & 1.103 & 1.022 & 1.197 & 1.029 & 1.024 & 1.027 & 1.014 \\
\hline$A R(A I C)-$ Trunc. & 0.925 & 0.919 & 0.919 & 0.971 & 0.963 & 0.962 & 0.968 & 0.984 \\
\hline$A R(P)-$ Trunc. & 1.108 & 1.048 & 0.986 & 1.131 & 1.023 & 1.018 & 1.018 & 1.006 \\
\hline$A R(A I C)-T S F$ & 0.901 & 0.938 & 1.011 & 0.956 & 0.944 & 0.940 & 0.948 & 0.982 \\
\hline \multirow{2}{*}{$A R(P)-T S F$} & 1.031 & 1.023 & 1.001 & 1.020 & 0.998 & 0.984 & 0.987 & 0.988 \\
\hline & \multicolumn{4}{|c|}{ Series 7} & & & & \\
\hline$A R(A I C)$ & 0.936 & 0.891 & 0.867 & 0.908 & & & & \\
\hline$A R(P)$ & 0.936 & 0.889 & 0.859 & 0.897 & & & & \\
\hline$A R(A I C)-$ Trunc. & 0.932 & 0.884 & 0.854 & 0.896 & & & & \\
\hline$A R(P)-$ Trunc. & 0.933 & 0.884 & 0.853 & 0.893 & & & & \\
\hline$A R(A I C)-T S F$ & 0.919 & 0.880 & 0.967 & 1.645 & & & & \\
\hline$A R(P)-T S F$ & 0.920 & 0.879 & 0.960 & 1.633 & & & & \\
\hline
\end{tabular}

Series 1: NAPM Production Index, Series 2: Employees on Non-farm Payrolls - Total Private, Series 3: Employee Hours in Non-ag. establishments (AR, bil. hours), Series 4: NAPM New Orders Index, Series 5: CPI Germany, Series 6: M1 UK, Series 7: Seasonally Adjusted Monthly Temperatures for the Northern Hemisphere. 